\title{
Utilisation des ressources en eau dans un contexte de changement climatique : réalités écologiques, sociales et économiques dans les communes Ambositra I et Ambositra II Madagascar
}

\author{
Rindramampionona Fanambinantsoa Ankasitrahana Randriamifidison ${ }^{1}$, Daniel Rakotoarisoa ${ }^{1,2}$, \\ Tsaralaza Jorlin Tsiavahananahary ${ }^{3}$, Tantely Rakotovao ${ }^{4}$, and Lily Arson Rene de Rolland ${ }^{5}$ \\ ${ }^{1}$ Institut Supérieur de Technologie d'Ambositra, Ambositra 306, Madagascar \\ ${ }^{2}$ Laboratoire Engénierie et Géosciences, Ecole Supérieure Polytechnique, Antananarivo 101, Madagascar \\ ${ }^{3}$ Faculté des Sciences de Technologie et Environnement, Université de Mahajanga, \\ Mahajanga 501, Madagascar \\ ${ }^{4}$ Direction de l'Energie de 1'Eau et des Hydrocarbures Amoron'i Mania, Ambositra 306, Madagascar \\ ${ }^{5}$ The peregrine Fund, Antananarivo 101, Madagascar \\ Correspondence: Rindramampionona Fanambinantsoa Ankasitrahana Randriamifidison \\ (rindrarandriamifidison@yahoo.fr)
}

Published: 16 November 2021

\begin{abstract}
Résumé. Les démarches de cette recherche ont été fondées au départ sur le fait que les ressources en eau diminuent avec le changement climatique, alors que les besoins de la population augmentent incéssament. Elle concerne la Commune urbaine d'Ambositra et la Commune rurale d'Ambositra, traversées par la rivière Isaha . Dans cette optique, cet article a pour objectif d'analyser le rapport entre les ressources et les besoins en eau pour diverses utilisateurs tenant compte des variabilités climatiques dans la zone. Pour ce faire, des variables d'entrée ont été utilisés pour modéliser de l'utilisation des ressources en eau par le biais du logiciel WEAP (Water Evaluation and Planning System), précédé par un traitement cartographique pour la délimitation de la zone par le logiciel QGIS. Les données sur la précipitation sont obtenues avec la médiane des valeurs issues des modèles climatiques du scenario RCP4.5. Les principaux résultats de cette étude concernent à la fois l'utilisation de l'eau à des fins agricoles et pour un usage urbain. Et les stratégies proposées concernent tous les acteurs de tous les niveaux, des utilisateurs à l'Etat central. Nous avons évoqué l'urgence de la concrétisation des recommandations émanant de cette étude vue les réalités actuelles ainsi que la prévision issue de la modélisation.
\end{abstract}

\section{Introduction}

La disponibilité en eau conditionne l'occupation humaine et toutes activités anthropiques. Cette ressource est devenue rare dans certains endroits à Madagascar (FAO, 2019). Cette étude a été conduite dans la zone d'Ambositra, située sur les hautes terres centrales de Madagascar, dans la Région Amoron'i Mania qui littéralement signifie au bord de la Mania, une grande rivière qui coule des montagnes centrales de Madagascar et qui se déverse dans le Canal de Mozambique en passant par le fleuve Tsiribihina (Chaperon et al., 1993). À Ambositra, la gestion de l'eau ne se fait pas d'une façon rationnelle. Traversé par la Route Nationale 7, Ambositra est une zone des hautes terres centrales de Madagascar. Une ville urbaine où règne toutefois un aspect rural. L'accès à l'eau potable y est encore très faible. Les contextes écologiques, économiques et sociales sont difficilement mâ̂trisables car ni la population, ni les autorités concernées ne sont assez conscientes de la réalité sur l'enjeu de cette ressource. Ce qui est à l'origine de cette recherche. Qui s'est proposée de (1) établir une base de donnée sur les ressources en eau disponibles selon les variabilités climatiques et sai- 
sonnières, (2) identifier, une typologie des principaux utilisateurs, et (3) proposer des stratégies adaptées aux réalités du site et aux paramètres de changement climatique

\section{Matériels et méthodes}

\subsection{Présentation de la zone d'étude}

Les deux Communes Ambositra I, Ambositra II se situent administrativement dans le District d'Ambositra, Région Amoron'i Mania; la ville d'Ambositra étant le Chef Lieu de la Région Amoron'i Mania (Fig. 1), et se situe à une distance de $255 \mathrm{~km}$ de la Capitale de Madagascar en empruntant la RN7 vers le sud. La population d'Ambositra compte 33276 habitants (INSTAT, 2019). Une espèce de plante actuellement disparue nommée vernaculairement «Somangana » dominait ces habitats ripicoles (Randriamifidison, 2015), ce qui était à l'origine de l'appellation plus ancienne et autochtone de Sahasomangana, actuellement Isaha; avec Sahasomangana iray sur la partie sud de la ville (iray pour dire en amont) et Sahasomangana iava sur la partie nord de la ville (iava pour dire en aval).

\subsection{La rivière Isaha}

L'hydrologie de la Région Amoron'i Mania est dominée par le bassin versant de la Tsiribihina. La Fig. 2 montre le réseau hydrographique de la Région. Le réseau hydrologique de ce bassin versant prend sa source dans la région et se déverse dans le Canal de Mozambique, une fois récupéré par le fleuve Tsiribihina (Chaperon et al., 1993). La rivière de l'Isaha est un plan d'eau permanent issu du fleuve Mania.

\subsection{Méthodes}

Une descente sur terrain a été effectuée pour une observation de la hauteur d'eau dans la rivière en période d'étiage (octobre 2019). En effet, en référence avec la démarche méthodologique de Rakotoarisoa (2018) et Rakotondrabe (2007) l'apport en eau de surface disponible à été obtenue. Elle est considérée comme variable d'entrée dans le modèle.

Dans cette modèle, la priorité de la demande représente la priorité de l'allocation d'une ressource limitée entre plusieurs sites de demande. Pour cette étude, deux politiques de répartition seront étudiées pour chaque scénario :

- La politique 1 priorisant les demandes de la population, besoins en eau pour les infrastructures sanitaire et éducatif (Code de l'Eau de Madagascar, 1999);

- La politique 2 favorisant les besoins en eau pour le secteur Agricole (agriculture et élevages).
Dans cette recherche, la chaîne de modélisation intégrative de la balance demande et offre en eau avec WEAP ${ }^{1}$ (Rakotoarisoa, 2018) a été adoptée et présentée dans la Fig. 3. DEP : demande en eau par la population; DEinf-San : demande en eau par l'infrastructure sanitaire; Deinf-Ed : demande en eau par l'infrastructure éducative; DE-agr : demande en eau par l'agriculture DE-élg : demande en eau par l'elevageLe processus de création de scenarii se déroule comme suit :

- Création de la zone d'étude : il s'agit d'ajouter la carte de la zone d'étude sous forme vecteur.

- Réglage de paramètres généraux : Dans cette étude, l'année 1991 a été choisie comme l'année de base de modélisation en limitant toutes les informations sur le système introduit dans le compte actuel entre la période 1991-2018.

- Création de site de demande : après avoir numérisé la rivière de l'Isaha; deux sites de demande ((Populations, agricultures) ont été créés.

- Création des hypothèses clés : ils sont des variables qui permettent de distinguer les scénarios proprement dits (climat variable, accroissement de population) aux scénarii de référence (situation imaginaire sans aucune variation interannuelle). Dans cet article, deux hypothèses clés ont été adoptées, le besoin de la population varie avec la croissance démographique et la variabilité climatique affecte la disponibilité de ressources pour les besoins de la population et de l'agriculture.

- Création de Scénarii : ils sont des variables construites à partir des hypothèses clés sur la base de l'état actuel. Trois scenarii ont été choisis dans cette étude pour la modélisation et comptée à partir de l'année 1991 jusqu'en 2050 (fin du scénario). : scenario de référence pris comme base avec une croissance continue de la population de $3.4 \%$; le deuxième scénario considère un fort accroissement de population (5\%); et le troisième scénario représente la variation du climat.

Pour le scénario «Changement climatique », il a été attribué aux régimes climatiques (très $\mathrm{sec}, \mathrm{sec}$, normale, humide, très humide) des valeurs numériques (Tableau 1) pour que le logiciel puisse les reconnaître.

Le type de climat est défini à partir de la précipitation annuelle reçue par la zone d'étude. L'attribution des régimes climatiques pour chaque année a été basée sur l'analyse des données climatiques (précipitation moyenne mensuelle) issues du portail du World Bank Group sur le climat ${ }^{2}$ selon les

1. Water Evaluation And Planning, est un outil convivial qui adopte une approche integrée dans la planification des ressources en eauUniversité de Stockholm.

2. https://climateknowledgeportal.worldbank.org/ download-data (la date du dernier accès : 18 octobre 2021). 

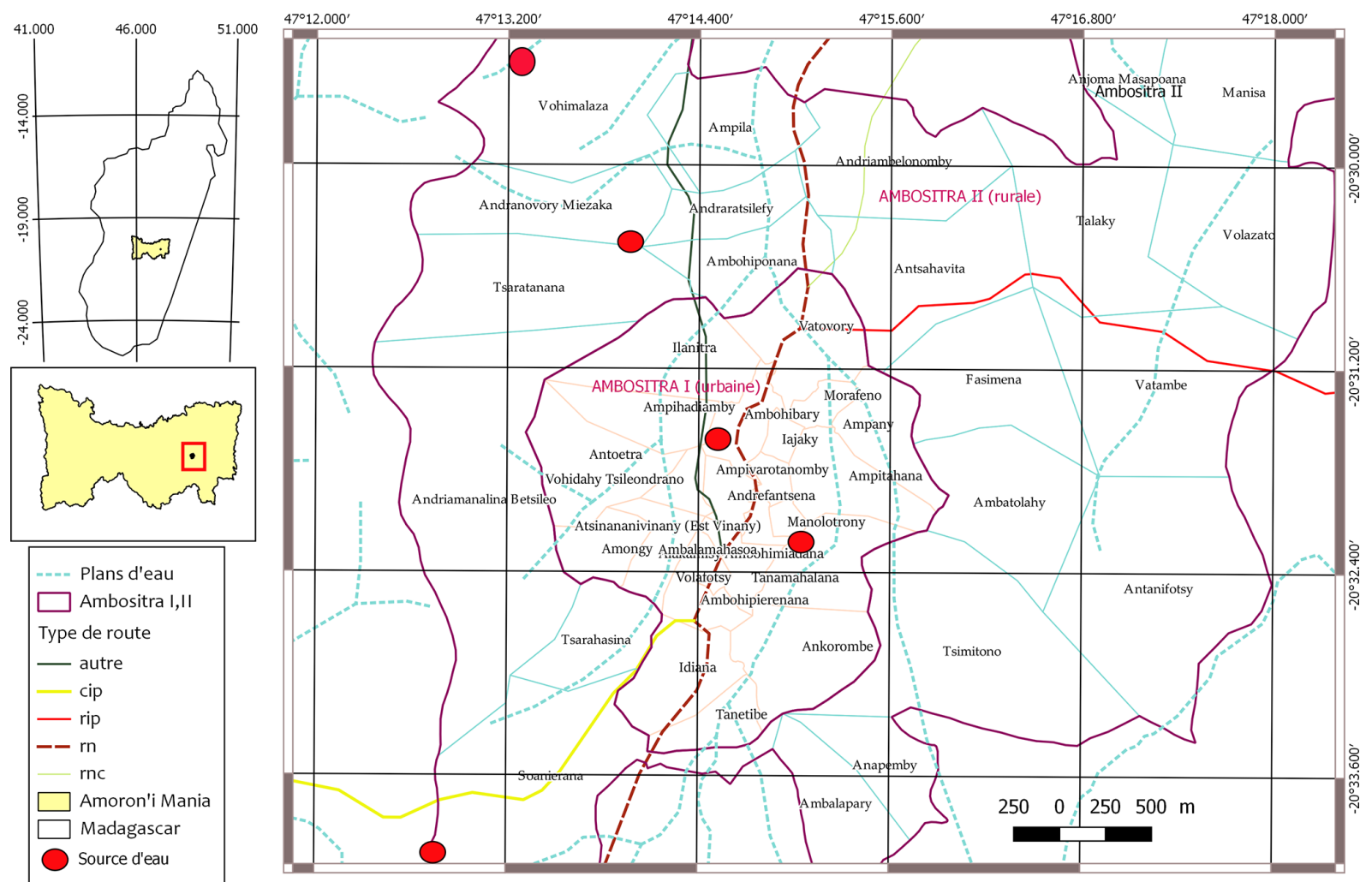

Source : INSTAT 2018, BD100 FTM Réalisation : Randriamifidison, 2020

Figure 1. Carte de localisation de la zone d'étude (Réalisation : R. F. A - Rindramampionona Fanambinantsoa Ankasitrahana).

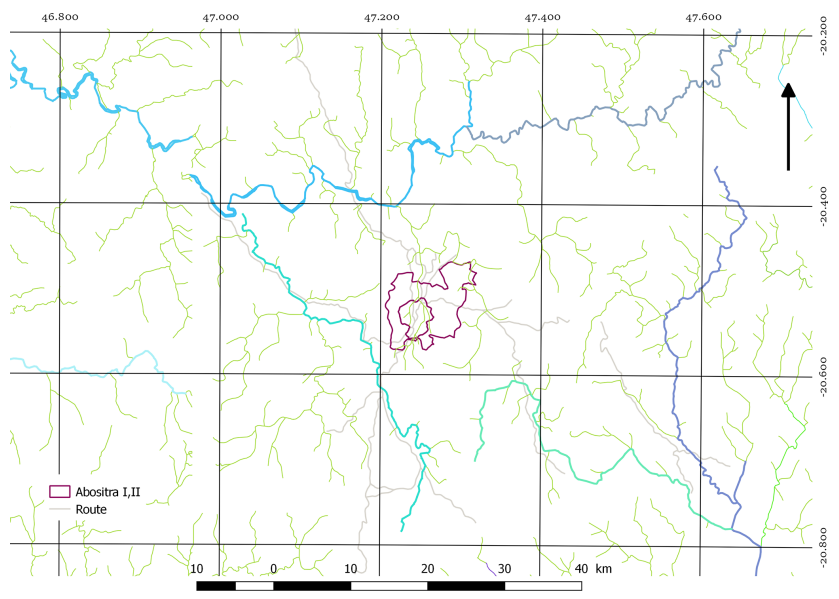

Figure 2. Aperçu hydrographique du site d'étude (Source : BD 100 FTM, Realisation : Randriamifidison, 2019).

modèles climatiques avec le scénarion RCP4.5. Ainsi nous avons adopté ces figures (Figs. 4 et 5) pour classifier les années.

La valeur moyenne correspond à la valeur de référence «normal » puis en dessous « sec » et « très sec », et au dessus « humide » et « très humide ».

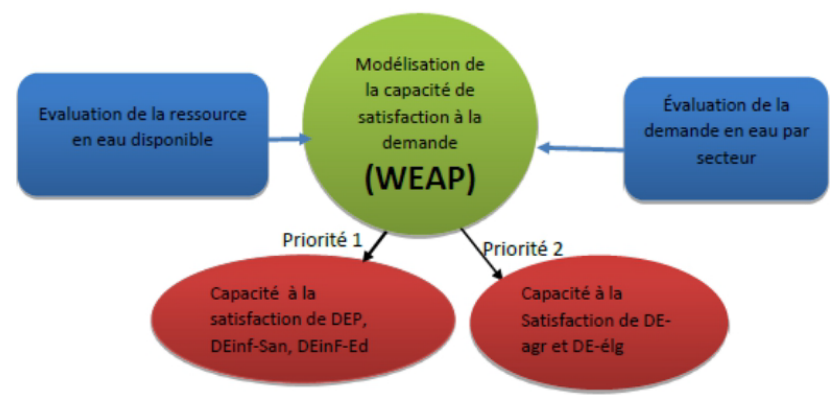

Figure 3. Modélisation avec le logiciel WEAP (Rakotoarisoa, 2018).

Ce qui nous amène à cette Fig. 6 .

\section{Résultats}

\subsection{Accès aux ressources en eau pour la population à Ambositra}

Pour Ambositra urbaine, la distribution de l'eau domestique est assurée par la JIRAMA (Jiro sy Rano Malagasy ou Electricité et Eau de Madagascar). Toutefois, ce n'est pas accessible à tous. Ambositra II, dispose de quelques ressources 
Tableau 1. Valeurs numériques relatives à chaque type de climat.

\begin{tabular}{lr}
\hline Types de climat & Valeurs correspondantes \\
\hline Très sec & 0.7 \\
Sec & 0.8 \\
Normale & 1 \\
Humide & 1.3 \\
Très humide & 1.45 \\
\hline
\end{tabular}

Source : Rakotondrabe (2007) et Rakotoarisoa (2018).

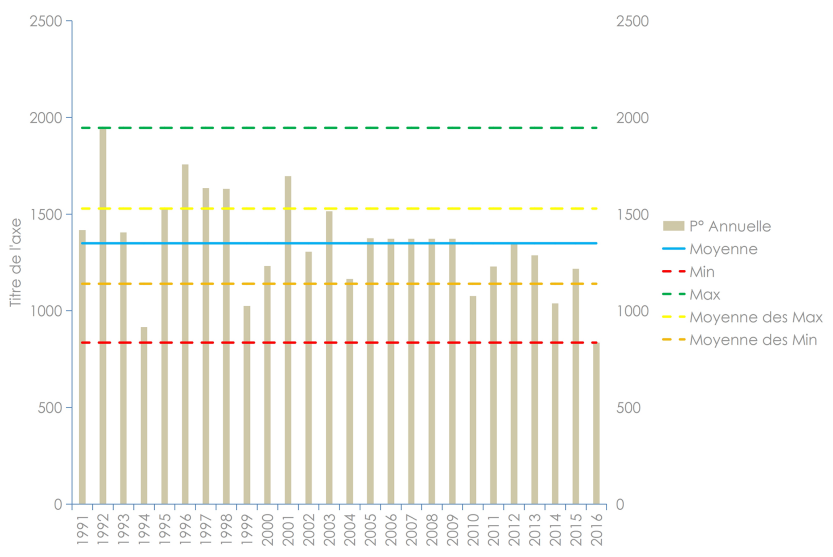

Figure 4. Précipitation annuelle depuis 1991 à 2015 (accessible à l'adresse suivante : https://climateknowledgeportal.worldbank.org/ download-data, la date du dernier accès : 18 octobre 2021).

dont le captage d'Ambositra I par la JIRAMA. Actuellement, le captage se situe dans la Commune Rurale d'Ankazoambo, la source d'Anasana, au sud est de la ville.

Généralement, d'après les observations sur sites, toutes les sources hydrologiques de surfaces (rivières et fleuves) sont de nature simple et de types permanent et dont la largeur ne dépasse pas $10 \mathrm{~m}$. Treize (13) sources hydrologiques sont répertoriées dont 3 sont de types temporaires. La production annuelle en eau potable par la JIRAMA est estimée en moyenne à $68823 \mathrm{~m}^{3}$, dont $65088 \mathrm{~m}^{3}$ utilisée (https://www.jirama.mg/ (la date du dernier accès : 28 octobre 2021), avec 2031 abonnés. Par rapport au nombre de population, ce chiffre est trop faible, moins de $3 \%$ de la population sont abonnés à l'eau de la JIRAMA, seul distributeur.

Les figures suivant montrent la répartition de la population par types de ressources (Figs. 7 et 8).

Ces figures montrent seulement la provenance des eaux domestiques, et pas les moyens d'accès. Les « Non répertoriés » selon la Direction de l'eau et de l'assainissement de la Région Amoron'i Mania constituent $75 \%$ de la population urbaine. Ils sont des abonnés de la JIRAMA (3\%) et le reste $(97 \%)$ utilise les bornes fontaines publiques pour leur usage domestique.

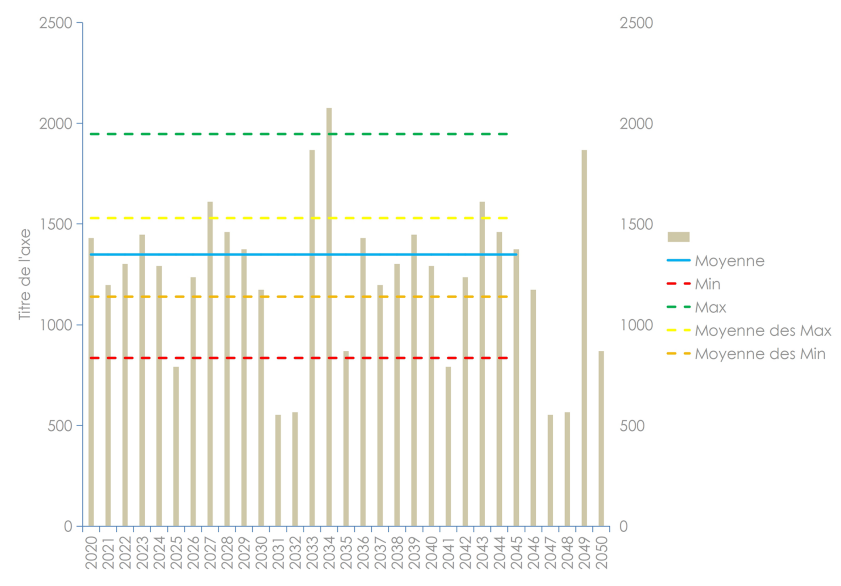

Figure 5. Précipitation annuelle depuis 2020 à 2050 (accessible à l'adresse suivante : https://climateknowledgeportal.worldbank.org/ download-data, la date du dernier accès : 18 octobre 2021).

\section{2 Évaluation quantitative de l'utilisation de l'eau}

Au vu des données de la Fig. 9, force est d'admettre que la ressource en eau est largement suffisante pour Ambositra, la demande est satisfaite (Demande non satisfaisante $=0 \mathrm{~m}^{3}$ ). Prenons en exemple pour le mois de Novembre (période de repiquage pendant laquelle le besoin en eau est le plus important pour l'agriculture), l'eau disponible est estimée à 8.9 millions de $\mathrm{m}^{3}$, alors que l'agriculture n'utilisent que 1.63 millions de $\mathrm{m}^{3}$.

Ces données semblent excellentes, mais la réalité n'est pas le cas. Ce qui nécessite l'analyse de l'aspect socioéconomique dans la partie discussion.

\subsection{Modélisation de l'utilisation de l'eau à l'horizon de 2050}

La Fig. 11 donne la variation de la quantité de l'eau de surface qui n'est pas utilisée ni par la population, ni par l'agriculture. Comme la population a été priorisé dans l'analyse, la valeur du débit de retour (quantité non consommée après utilisation) étant nulle, donc seule l'utilisation à des fins agricoles présentent une quantité très importante d'eau non utilisée. La Fig. 12 qui suit montre la situation en 2050, avec les scenarii d'une forte croissance démographique de l'ordre de $5 \%$ ainsi que des séquences hydrologiques sèches prolongées. Rappelons que la projection de la précipitation pour 2030 donne une baisse en moyenne mensuelle de la précipitation allant de 112.38 à $105.45 \mathrm{~mm}$ $(-6.92 \mathrm{~mm})$ (Randriamifidison, 2019). A partir de cette figure, il est démontré une augmentation des besoins totaux (population + agriculture) à partir de 2017-2018. Des variations mensuelles sont constatées, justifiés par une variabilité climatique qui perturbe les calendriers culturaux, dont les besoins en eau. 


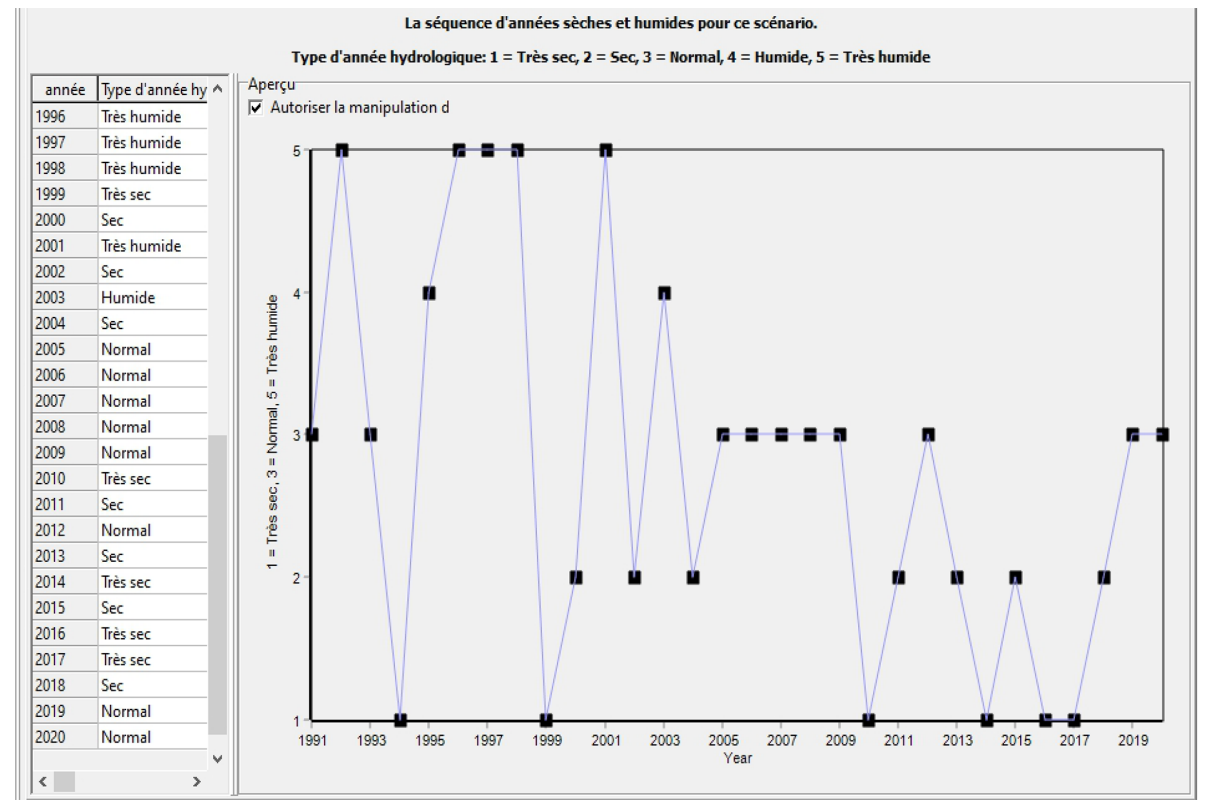

Figure 6. Attributions de séquences d'année sèches et humides pour identifier le scénario lié à la précipitation dans WEAP.

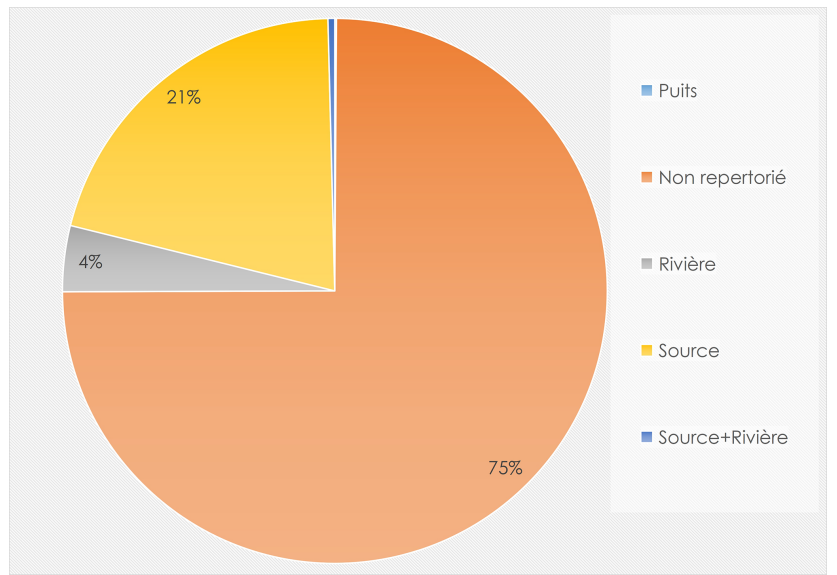

Figure 7. Répartition de la population par type de ressources dans la Commune Urbaine d'Ambositra (Direction de l'Eau Amoron'i Mania, données non publiées).

\section{Discussion et recommandations}

La pauvreté de la population affecte les moyens d'accès à l'eau potable (traitée). La plupart des ménages urbains sont en location d'appartement et n'envisage pas de s'abonner à la JIRAMA n'étant pas propriétaire. Pour y remédier, exiger une installation pour l'accès à l'eau courante pour chaque nouvelle construction, augmenter le nombre de bornes fontaines ou des puits à pompages manuelles ou électrique solaire au niveau de chaque quartier. Pour la zone rurale, l'utilisation de la source doit être améliorée, en ramenant l'eau par conduite forcée au village ou en aménageant le site et ses

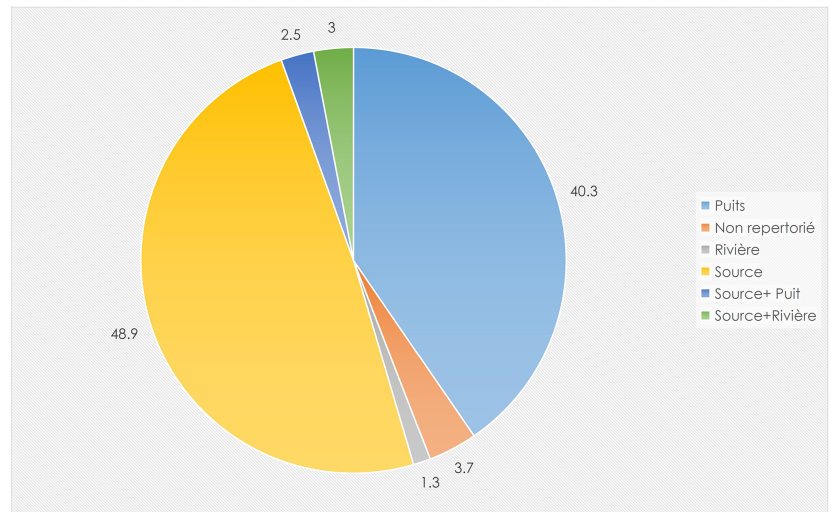

Figure 8. Répartition de la population par type de ressources dans la Commune Urbaine Rurale d'Ambositra (Direction de l'Eau Amoron'i Mania, données non publiées).

alentours pour le respect de l'hygiène et pour pérenniser la source, renforcer le reboisement avec des espèces à racines adventives pour faciliter l'infiltration et entretenir la nappe phréatique.

Les ressources en eau peuvent satisfaire les besoins de la population, toutefois, des problèmes d'irrigation se font ressentir de plus en plus au niveau des rizières de part et d'autres de la rivière Isaha. Cela résulte d'une mauvaise gestion de l'eau, le calendrier cultural est perturbé avec les variabilités du climat. Ce problème devient même une source de litige entre les paysans. Car l'eau peut être bloqué par un riziculteur en amont. Cela pourrait être résolu par la mise en place d'une association des usagers de l'eau pour respecter les exigences communes. La mise en place d'un bassin de décan- 


\begin{tabular}{|c|c|c|c|c|c|c|c|c|c|c|c|c|c|}
\hline & Jan & Fév & Mars & Avr & Mai & Juin & Juil & Août & Sept & Oct & Nov & Déc & Sum \\
\hline \multicolumn{14}{|c|}{ Débits entrant dans l'aire (Million Mètre cube) } \\
\hline En dessous de IsahaDébit de tête & 29,38 & 26,47 & 29,73 & 16,30 & 9,91 & 6,74 & 6,43 & 5,92 & 4,38 & 4,18 & 8,09 & 22,42 & 169,95 \\
\hline \multicolumn{14}{|c|}{ Débits sortant de l'aire (Million Mètre cube) } \\
\hline Demande agri & 0,00 & 0,10 & 0,18 & 0,20 & 0,00 & 0,00 & 1,10 & 1,97 & 1,11 & 1,19 & 1,63 & 1,30 & 8,78 \\
\hline Demande pop & 0,00 & 0,00 & 0,00 & 0,00 & 0,00 & 0,00 & 0,00 & 0,00 & 0,00 & 0,00 & 0,00 & 0,00 & 0,00 \\
\hline En dessous de Demande agri Retour & 29,38 & 26,37 & 29,55 & 16,10 & 9,91 & 6,74 & 5,33 & 3,95 & 3,27 & 2,99 & 6,46 & 21,12 & 161,17 \\
\hline Sum & 29,38 & 26,47 & 29,73 & 16,30 & 9,91 & 6,74 & 6,43 & 5,92 & 4,38 & 4,18 & 8,09 & 22,42 & 169,95 \\
\hline \multicolumn{14}{|l|}{ Demande non satisfaite [Mètre cube] } \\
\hline Demande agri & 0,00 & 0,00 & 0,00 & 0,00 & 0,00 & 0,00 & 0,00 & 0,00 & 0,00 & 0,00 & 0,00 & 0,00 & 0,00 \\
\hline Demande pop & 0,00 & 0,00 & 0,00 & 0,00 & 0,00 & 0,00 & 0,00 & 0,00 & 0,00 & 0,00 & 0,00 & 0,00 & 0,00 \\
\hline Sum & 0,00 & 0,00 & 0,00 & 0,00 & 0,00 & 0,00 & 0,00 & 0,00 & 0,00 & 0,00 & 0,00 & 0,00 & 0,00 \\
\hline \multicolumn{14}{|c|}{ Exigence distribution (avec pertes, recycl., GSD) (Million Mètre cube) } \\
\hline Demande agri & 0,00 & 0,17 & 0,30 & 0,33 & 0,00 & 0,00 & 1,83 & 3,29 & 1,85 & 1,98 & 2,71 & 2,17 & 14,64 \\
\hline Demande pop & 0,00 & 0,00 & 0,00 & 0,00 & 0,00 & 0,00 & 0,00 & 0,00 & 0,00 & 0,00 & 0,00 & 0,00 & 0,00 \\
\hline Sum & 0,00 & 0,17 & 0,30 & 0,33 & 0,00 & 0,00 & 1,83 & 3,29 & 1,85 & 1,98 & 2,71 & 2,17 & 14,64 \\
\hline
\end{tabular}

Figure 9. Données de référence moyennes mensuelles sur l'eau disponible et utilisée (Source : Modélisation avec WEAP).

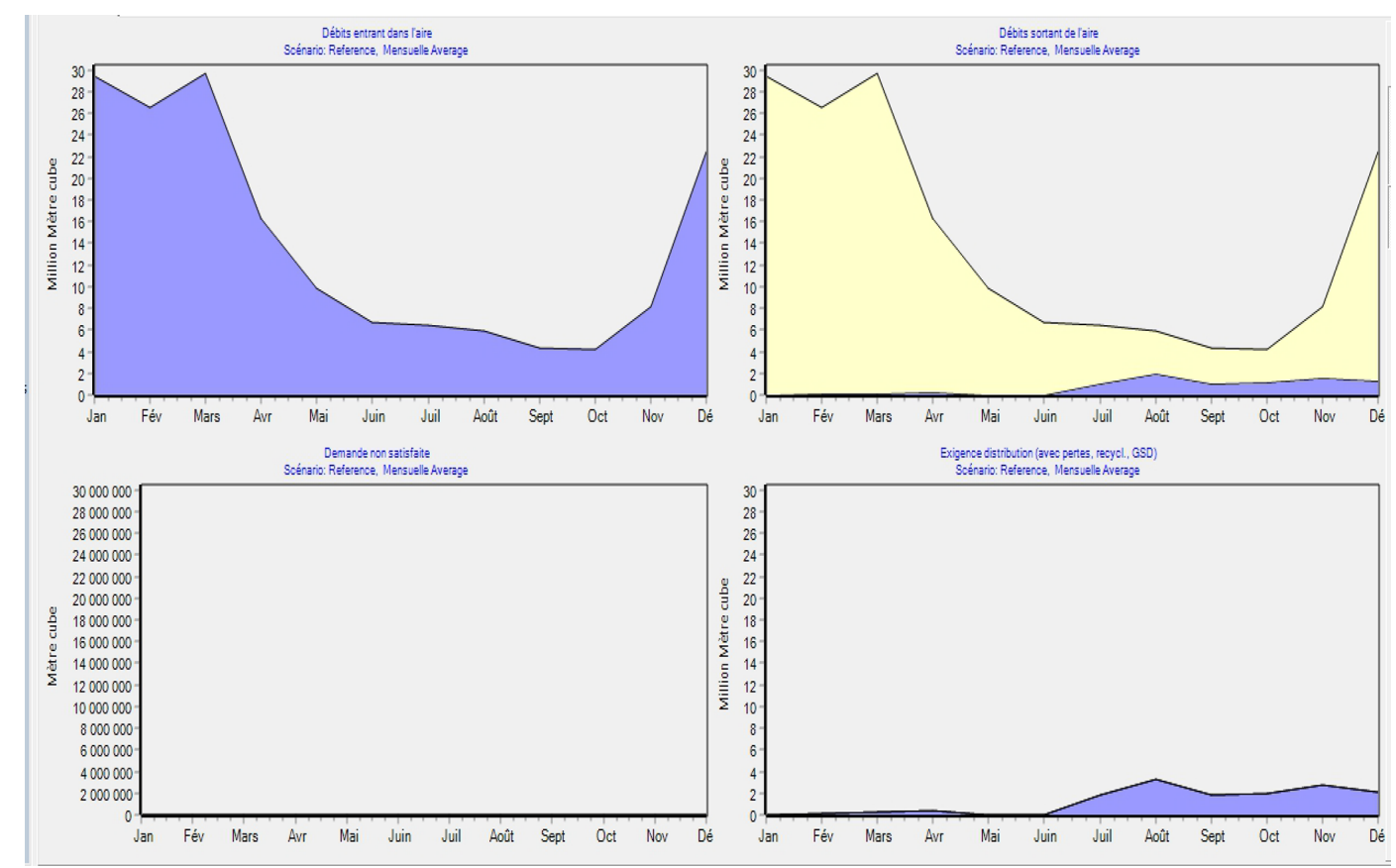

Figure 10. Débit entrant, débit sortant, demande non satisfaisante, exigence de distribution (Modélisation avec WEAP).

tation qui servira de stockage de l'eau de pluies au moment des crues pour l'utiliser après pour irriguer les rizières et les cultures vivrières. Promouvoir la riziculture pluvial. Et modifier la perception de la disponibilité en eau comme disponibilité de pluie.

\section{Conclusion}

Les ressources en eau de surfaces à Ambositra sont satisfaisantes, du point de vue quantitative. Cependant, tenant compte des réalités socio-économique et écologiques, il a été soulevé que la plupart de la population n'ont accès à l'eau potable au sein de leur habitations. Par ailleurs, la plupart de cette quantité élevée devient des eaux de ruissellement engendrant sur leur passage des érosions très fréquentes sur les 


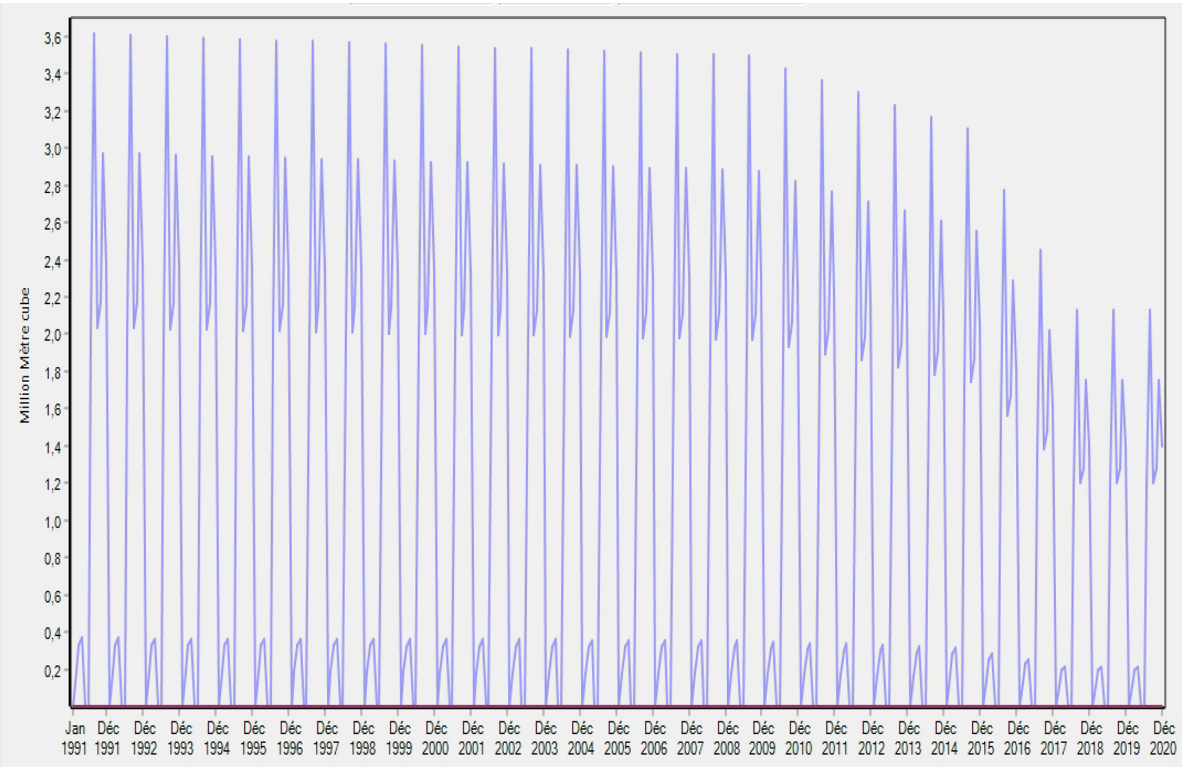

Figure 11. Débit de retour (non utilisée) (Modélisation avec WEAP).

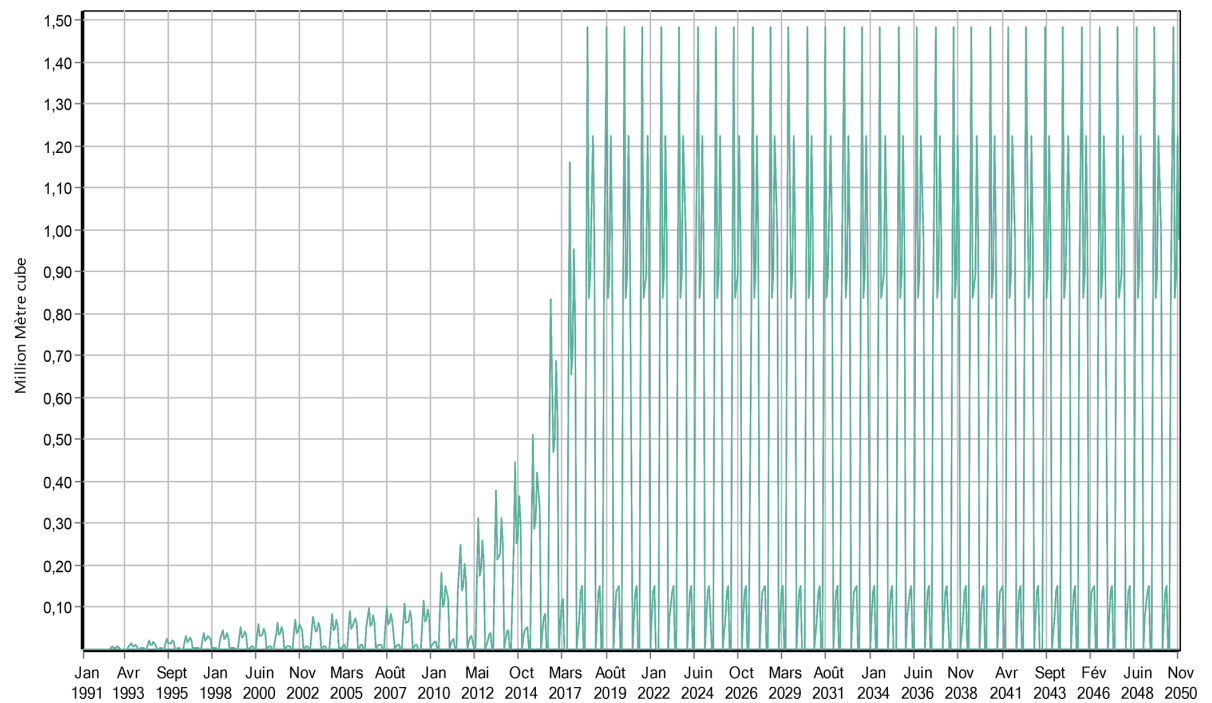

Figure 12. Modélisation de l'utilisation mensuelle de l'eau jusqu'en 2050 (Modélisation avec WEAP).

Hautes Terres de Madagascar. Ceci, du fait que la végétation ait disparu au profit des terrains dénudés, infertiles et hostiles à l'agriculture dans plusieurs cas, laissant place aux tanety ${ }^{3}$ plein de lavakas ${ }^{4}$. Il n'est pas alors tard pour remettre en question les pratiques ancestrales et penser à la pérennisation de l'utilisation, en respect du principe d'équitabilité sociale et économique du développement durable, tout en tenant compte que sans couverture végétale, l'eau ne pourra être retenue. Cette étude est loin d'être complète, toutefois,

3. Vaste étendue de collines dénudéss.

4. Fissures, grands trous causés par une forte érosion sur un tanety. elle ouvre une perspective assez large d'approfondissement de l'aspect des eaux souterraines, de la capacité d'infiltration, pour la résilience face aux crues et sécheresses.

Disponibilité du code et des données. Codes and data are available upon request by contacting the correspondence author.

Collaborateurs. RFAR et DR ont assuré la conceptualisation, le montage du protocole expérimental ainsi que la redaction de l'article. TJT a apporté sa contribution dans les simulations. TR était l'administrateur du projet, LARDR pour la supervision. 
Intérêts concurrents. Les auteurs déclarent qu'ils n'ont aucun conflit d'intérêts.

Clause de non-responsabilité. Publisher's note : Copernicus Publications remains neutral with regard to jurisdictional claims in published maps and institutional affiliations.

Déclaration du numéro spécial. This article is part of the special issue "Hydrology of Large River Basins of Africa". It is a result of the 4th International Conference on the "Hydrology of the Great Rivers of Africa", Cotonou, Benin, 13-20 November 2021.

Remerciements. We would like to thank all the co-authors, especially the Regional Director of Water, Sanitation and Hygiene of Amoron'i Mania. May this work find its place in the water management strategies of the Amoron'i Mania Region.

\section{Références}

Chaperon, P., Danloux, J., et Luc, F. : Fleuves et Rivières de Madagascar, Monographie hydrologique, IRD/DMH/CNREm IRD Éditions 2005, 548-557, accessible à l'adresse suivante : https://www.pseau.org/outils/ouvrages/ird_fleuves_et_ rivieres_de_madagascar_1993.pdf (la date du dernier accès : 26 octobre 2021), 1993.

Code de l'Eau de Madagascar : J. O. no. 2557 E.S. du 27.01.99, p. 735 , accessible à l'adresse suivante : http://www.droit-afrique. com/upload/doc/madagascar/Madagascar-Code-1999-Eau.pdf, (la date du dernier accès : 15 février 2020), 1999.
FAO : https://www.fao.org, la date du dernier accès : 20 Septembre 2019.

INSTAT : https://www.instat.mg (la date du dernier accès : 5 Janvier 2020), 2019.

Rakotoarisoa, D. : Gestion Integrée des Ressources en Eau du Bassin Versant de Soavina, Region Amoron'i Mania, Thèse de doctorat en Génie minéral et pétrolier, Ecole doctorale Ingénierie et Géosciences, ESPA, Université d'Antananarivo, Antananarivo, p. 46, 2018.

Rakotoarisoa, D., Rasolomanana, J., Ralaimaro, J., Andrimanaosoa, N., Rakoto, H., et Mandimbiharison, A. : Gestion intégrée des ressources en eau du Bassin versant de Soavina par télédétection et SIG, Région Amoron'i Mania Fianarantsoa, Revues Madamines, volume 1, ISSN 2220-068, accessible à l'adresse suivante : http ://madarevues.recherches.gov.mg/ ?Gestion-integreedes-ressources-en-eau-du-Bassin-versant (la date du dernier accès : 26 octobre 2021), 2018.

Rakotondrabe, F. : Etude de la vulnérabilité des ressources en eau aux changements climatiques, modélisation par le logiciel WEAP 21 : cas du bassin versant de Morondava (Sud-ouest de Madagascar), Mémoire de recherches pour l'obtention du diplôme d'etudes approfondies spécialisation : hydrogeologie, 7083, accessible à l'adresse suivante : https://www.weap21.org/ downloads/Madagascar.pdf (la date du dernier accès : 26 octobre 2021), 2007.

Randriamifidison, R. F. A., Rakotoarisoa, D., Tsiavahananahary, T. J., and Andriamampihantona, F. : La Région Amoron'i Mania face aux changements climatques : realités, adaptation, projection et orientations strategiques pour un développement durable, Acte du 32 ème Colloque de l'Association Internationale de Climatologie 2019, 45-50, accessible à l'adresse suivante : http://www.climato.be/aic/colloques/actes/ Thessaloniki2019_actes.pdf (la date du dernier accès : 18 octobre 2021), 2019. 